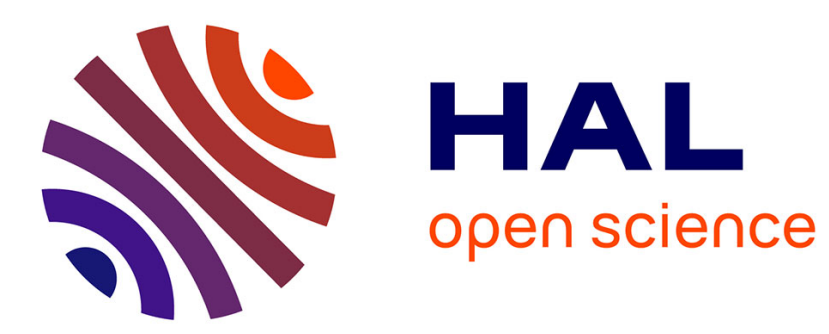

\title{
Polynomial programming prevents aircraft (and other) conflicts
}

\author{
Martina Cerulli, Leo Liberti
}

\section{To cite this version:}

Martina Cerulli, Leo Liberti. Polynomial programming prevents aircraft (and other) conflicts. Operations Research Letters, 2021, 10.1016/j.orl.2021.05.001 . hal-02971109v2

\section{HAL Id: hal-02971109 \\ https://hal.science/hal-02971109v2}

Submitted on 12 Feb 2021

HAL is a multi-disciplinary open access archive for the deposit and dissemination of scientific research documents, whether they are published or not. The documents may come from teaching and research institutions in France or abroad, or from public or private research centers.
L'archive ouverte pluridisciplinaire HAL, est destinée au dépôt et à la diffusion de documents scientifiques de niveau recherche, publiés ou non, émanant des établissements d'enseignement et de recherche français ou étrangers, des laboratoires publics ou privés. 


\title{
Polynomial programming prevents aircraft (and other) conflicts
}

\author{
Martina Cerulli, Leo Liberti \\ LIX - CNRS, École Polytechnique, Institut Polytechnique de Paris, Palaiseau, 91128,France
}

\begin{abstract}
Using a known algebraic result, we obtain a finite polynomial programming reformulation of a semi-infinite program modeling the aircraft deconfliction problem via subliminal speed regulation. Solving the reformulation often yields better results than the state of the art.
\end{abstract}

Keywords: semi-infinite programming, quadratic programming, aircraft deconfliction, distance constraint

\section{Introduction}

In air traffic management, the aircraft deconfliction problem (ADP) aims at ensuring the respect of a required distance among flying aircraft, while optimizing a certain objective function. Several strategies are available to pursue this goal: changing aircraft altitudes, heading angles, or speed. All of them are mainly implemented by human air traffic controllers (ATC) who are in charge of detecting and solving potential conflicts among aircraft flying in a restricted airspace.

Nowadays, given the progressive increase in air traffic, it is increasingly interesting to introduce automation in aircraft deconfliction, as well as in urban air mobility. The speed regulation (SR) strategy, in particular, has been studied in the context of the European project ERASMUS [1], which introduced the concept of subliminal speed control. This consists in slightly modifying aircraft speed in an imperceptible way for ATC, but in such a way that the number of conflicts is reduced upstream of the control, thus reducing ATC's workload.

In this paper, we focus on ADP via subliminal SR and formulate it using Semi-Infinite Programming (SIP). In order to address the issue of uncountably many constraints, we reformulate it using Polynomial Programming (PP).

SR is one of the most used strategies for aircraft deconfliction by Mathematical Programming. In Section 4, we compare our approach with the one proposed in [2], where a Mixed Integer Nonlinear Programming (MINLP) formulation for ADP via SR in 2 dimen-

Email addresses: mcerulli@lix.polytechnique.fr (Martina Cerulli), liberti@lix.polytechnique.fr (Leo Liberti) 
sions is considered, and small instances of the problem are solved to global optimality by means of an existing exact solver, while for bigger instances a heuristic procedure (where the problem is decomposed and locally exactly solved) is proposed. In [3] another MINLP formulation of the same problem is proposed and solved using a feasibility pump heuristic. This algorithm, at each iteration, solves alternatively two subproblems obtained by two relaxations of the original problem, minimizing the distance between their solutions. In [4], two Mixed Integer Linear Programming formulations are presented: one using only speed changes, and the other on heading angles changes (HAC). Both [5] and [6] focus only on this last strategy. In particular, in [5] trajectories are modeled with B-splines and a SIP formulation of ADP via HAC is presented, reformulated with an exact penalty function, and solved using local optimization methods. A two-step approach is presented in [6]. The first step consists of a nonconvex MINLP model based on geometric constructions, which aims to minimize the weighted HAC to obtain the new conflict-free flight configuration. The second step consists of a set of unconstrained quadratic optimization models solved as a post-processing step to return each aircraft to its original flight plan as soon as possible after conflict resolution. In fact, SR strategy cannot to solve face-to-face conflict, and it may not be enough to guarantee safety if speed bounds are tight. That is why a lot of works in the literature focus on combined maneuvers too. For instance, in [7] a complex number formulation with disjunctive linear constraints is introduced to model ADP using both SR and HAC; different relaxations of the resulting MINLP are then proposed, solved, and compared.

The application scope of our new PP reformulation for SIP model of ADP extends to all application settings where distance constraints must be imposed at each of uncountably many time instants. We offer three examples. Trajectories in a fleet of underwater autonomous vehicles cannot come exceedingly close [8] during the time horizon of the operation. In reservoir engineering the (ramified) geometry of the underground pipes must be decided in such a way that branches from different wells are positioned at any point of a given trajectory depending on the ramification structure, but not too close to each other [9]. When we focus on a three-dimensional space, our approach is more relevant in the context of urban air mobility. In fact, Unmanned Aerial Vehicles have different dynamics w.r.t aircraft and the minimum distance between them can be ensured by using SR also during altitude changes (therefore the need of a third dimension to take into account). Values of $k$ larger than three may be useful in modelling other aircraft coordinates impacting the trajectory, such as load, engine power, and other controls; or fleets of objects with pairwise distances.

The fact that such SIP problems could be reformulated to PP ones (either via Lasserretype semidefinite relaxations [10] or kinetic distance matrices [11]) was previously known. Previous results, however, only offered relaxations, because of the large size and polynomial degree of the corresponding (nonconvex) formulations. In this paper, on the other hand, we provide a reasonably small PP formulation having the same polynomial degree as the original SIP problem, which can be solved in practice to derive feasible solutions.

The rest of this paper is organized as follows. In Section 2 we introduce the SIP formulation of the ADP via SR. In Section 3 we propose our new PP reformulation of 
the SIP problem. In Sect 4 we present some computational results showing the practical applicability of our PP reformulation.

\section{Semi-infinite formulation}

In this section we formulate the ADP via SR using Mathematical Programming. The decision variables quantify the speed changes. The objective function consists in minimizing the total speed changes. An uncountable set of constraints guarantee the safety distance on each pair of aircraft flying in the airspace considered during the given time horizon. The following natural formulation of the ADP was already presented in [12].

The index sets, parameters and decision variables are involved in all of the formulations presented in this paper (not only the SIP one). We remark that they are taken from [2].

\section{- Sets:}

- $A=\{1, \ldots, i, \ldots, n\}$ is the set of aircraft flying in a shared airspace;

$-K=\left\{1, \ldots, k_{\max }\right\}$ is the set of dimension indices.

\section{- Parameters:}

- $[0, T]$ is the time interval taken into account, with $\mathrm{T}$ expressed in hours;

$-d$ is the safety distance between aircraft [Nautical Miles NM, $1 \mathrm{NM}=1852 \mathrm{~m}$ ];

- $x_{i k}^{0}$ is the $k$-th component of the initial position of aircraft $i$;

$-v_{i}$ is the initially planned speed of aircraft $i[\mathrm{NM} / \mathrm{h}]$;

- $u_{i k}$ is the $k$-th component of the direction of aircraft $i$;

$-q_{i}^{\min }$ and $q_{i}^{\max }$ define the feasible range of the speed modification ratios of aircraft $i$ s.t. $q_{i}^{\min }<1<q_{i}^{\max }$.

- Variables: $q_{i}$ is the ratio of the implemented speed to the initially planned speed of aircraft $i: q_{i}=1$ if the speed is equal to the initially planned one, $q_{i}>1$ if it is increased, $q_{i}<1$ if it is decreased. We assume that $q_{i}$ is constant in the time interval considered, namely that each aircraft starts flying with the new implemented speed.

We now present objective function and constraints.

$$
\begin{array}{cl}
\min _{q^{\min } \leq q \leq q^{\max }} & \sum_{i \in A}\left(q_{i}-1\right)^{2} \\
\forall i<j \in A, \forall t \in[0, T] & \sum_{k \in K}\left[\left(x_{i k}^{0}-x_{j k}^{0}\right)+t\left(q_{i} v_{i} u_{i k}-q_{j} v_{j} u_{j k}\right)\right]^{2} \geq d^{2} .
\end{array}
$$


We remark that $(1 \mathrm{~b})$ contains uncountably many constraints quantified over $t$. For this reason formulation (1a)-(1b) is a SIP program. Constraints (1b) ensure aircraft separation requiring that the squared Euclidean distance between each pair of aircraft $(i, j)$ to be greater than or equal to $d^{2}$ at each instant $t$ in $[0, T]$.

The classical approach to solve SIP problem like the one above presented consists in discretizing with respect to the time $t$, i.e., replacing the set $[0, T]$ with a finite one and solving a sequence of finite programming problems, by applying appropriate linear or nonlinear programming algorithms,

\subsection{Polishing the polynomial}

For each $i<j \in A$, we define the polynomial:

$$
p_{i j}(t):=\sum_{k \in K}\left[\left(x_{i k}^{0}-x_{j k}^{0}\right)+t\left(q_{i} v_{i} u_{i k}-q_{j} v_{j} u_{j k}\right)\right]^{2}-d^{2}
$$

in function of $t$. We have:

$$
\begin{aligned}
p_{i j}(t)= & \sum_{k \in K}\left[\left(x_{i k}^{0}-x_{j k}^{0}\right)+t\left(q_{i} v_{i} u_{i k}-q_{j} v_{j} u_{j k}\right)\right]^{2}-d^{2} \\
= & \sum_{k \in K}\left[\left(x_{i k}^{0}-x_{j k}^{0}\right)^{2}+t^{2} q_{i}^{2}\left(v_{i} u_{i k}\right)^{2}+t^{2} q_{j}^{2}\left(v_{j} u_{j k}\right)^{2}-2 t^{2} v_{i} u_{i k} v_{j} u_{j k} q_{i} q_{j}\right. \\
& \left.+2 t\left(x_{i k}^{0}-x_{j k}^{0}\right)\left(v_{i} u_{i k}\right) q_{i}-2 t\left(x_{i k}^{0}-x_{j k}^{0}\right)\left(v_{j} u_{j k}\right) q_{j}\right]-d^{2} \\
= & \sum_{k \in K}\left(x_{i k}^{0}-x_{j k}^{0}\right)^{2}+t^{2} q_{i}^{2} \sum_{k \in K}\left(v_{i} u_{i k}\right)^{2}+t^{2} q_{j}^{2} \sum_{k \in K}\left(v_{j} u_{j k}\right)^{2}-2 t^{2} q_{i} q_{j} \sum_{k \in K}\left(v_{i} u_{i k} v_{j} u_{j k}\right) \\
& +2 t q_{i} \sum_{k \in K}\left(x_{i k}^{0}-x_{j k}^{0}\right)\left(v_{i} u_{i k}\right)-2 t q_{j} \sum_{k \in K}\left(x_{i k}^{0}-x_{j k}^{0}\right)\left(v_{j} u_{j k}\right)-d^{2} \\
= & \left(B_{i} q_{i}^{2}+B_{j} q_{j}^{2}-2 C_{i j} q_{i} q_{j}\right) t^{2}+2\left(D_{i j}^{i} q_{i}-D_{i j}^{j} q_{j}\right) t+A_{i j}-d^{2},
\end{aligned}
$$

where $A_{i j}, B_{i}, B_{j}, C_{i j}, D_{i j}^{i}, D_{i j}^{j}$ are constant (w.r.t. $t$ ) defined as follows:

$$
\begin{aligned}
A_{i j} & :=\sum_{k \in K}\left(x_{i k}^{0}-x_{j k}^{0}\right) & C_{i j} & :=\sum_{k \in K} v_{i} u_{i k} v_{j} u_{j k} \\
B_{i} & :=\sum_{k \in K}\left(v_{i} u_{i k}\right)^{2} & B_{j} & :=\sum_{k \in K}\left(v_{j} u_{j k}\right)^{2} \\
D_{i j}^{i} & :=\sum_{k \in K}\left(x_{i k}^{0}-x_{j k}^{0}\right)\left(v_{i} u_{i k}\right) & D_{i j}^{j} & :=\sum_{k \in K}\left(x_{i k}^{0}-x_{j k}^{0}\right)\left(v_{j} u_{j k}\right) .
\end{aligned}
$$

Thus,

$$
p_{i j}(t)=\left(B_{i} q_{i}^{2}+B_{j} q_{j}^{2}-2 C_{i j} q_{i} q_{j}\right) t^{2}+2\left(D_{i j}^{i} q_{i}-D_{i j}^{j} q_{j}\right) t+A_{i j}-d^{2}
$$

is a polynomial of second degree in $t$.

We can now rewrite the SIP formulation (1a)-(1b) as:

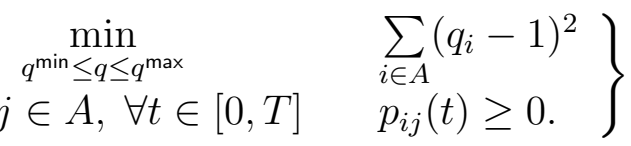

Probl. (3) is the minimization of $\sum_{i \in A}\left(q_{i}-1\right)^{2}$ subject to the second degree polynomial $p_{i j}(t)$ being non-negative on $t \in[0, T]$. 


\section{Reformulation to polynomial programming}

We introduce now a reformulation of (3) based on a result from [13]. This allows us to obtain a (finite) PP problem of the same degree of the original formulation (3).

In particular, the following proposition is an immediate corollary of [13, Lemma 2.1].

\subsection{Proposition (corollary of Lemma 2.1 from [13])}

For any $i<j \in A$, the polynomial $p_{i j}(t)$ is non-negative on $[0, T]$ iff there is a $2 \times 2$ positive semidefinite matrix

$$
M_{i j}=\left(\begin{array}{cc}
m_{i j} & r_{i j} \\
r_{i j} & g_{i j}
\end{array}\right) \succeq 0
$$

and a nonnegative scalar $\mu_{i j} \geq 0$ such that:

$$
p_{i j}(t)=(1 t) M_{i j}\left(\begin{array}{c}
1 \\
t
\end{array}\right)+(T-t) t \mu_{i j} .
$$

We use Proposition 3.1 to introduce an exact reformulation of the SIP Probl. (3), as shown in Theorem 3.1.

\subsection{Theorem}

The following formulation:

$$
\begin{aligned}
& \min _{M, \mu, q} \sum_{i \in A}\left(q_{i}-1\right)^{2} \\
& \text { s.t. } \quad g_{i j}-\mu_{i j}=B_{i} q_{i}^{2}+B_{j} q_{j}^{2}-2 C_{i j} q_{i} q_{j} \quad \forall i<j \in A \\
& 2 r_{i j}+T \mu_{i j}=2\left(D_{i j}^{i} q_{i}-D_{i j}^{j} q_{j}\right) \quad \forall i<j \in A \\
& m_{i j}=A_{i j}-d^{2} \\
& \left(r_{i j}\right)^{2} \leq m_{i j} g_{i j} \\
& \begin{aligned}
m_{i j}, g_{i j}, \mu_{i j} & \geq 0 \\
q^{\text {min }} \leq q & \leq q^{\max }
\end{aligned} \\
& \forall i<j \in A \\
& \forall i<j \in A \\
& \forall i<j \in A
\end{aligned}
$$

is an exact reformulation of (1a)-(1b).

Proof. Note that $p_{i j}(t)$ is given in two different forms in Eq. (2) and Eq. (4). We can therefore match coefficients of terms in $t$. This yields the following system:

$$
\begin{aligned}
g_{i j}-\mu_{i j} & =B_{i} q_{i}^{2}+B_{j} q_{j}^{2}-2 C_{i j} q_{i} q_{j} & & \forall i<j \in A \\
2 r_{i j}+T \mu_{i j} & =2\left(D_{i j}^{i} q_{i}-D_{i j}^{j} q_{j}\right) & & \forall i<j \in A \\
m_{i j} & =A_{i j}-d^{2} & & \forall i<j \in A,
\end{aligned}
$$

which is independent of $t$ by construction. We now have to impose the constraints $M_{i j} \succeq 0$ and $\mu_{i j} \geq 0$ given in the statement of Prop. 3.1. For the former, we observe that the $2 \times 2$ matrix $M_{i j}$ is positive semidefinite iff $\left(r_{i j}\right)^{2} \leq m_{i j} g_{i j}$ and $m_{i j}, g_{i j} \geq 0$, which yields the 
corresponding constraints in formulation (5). The latter is simply copied from formulation (3) to $(5)$.

We observe that Probl. (5) is a quadratic PP problem, and the degree is the same as in the original formulation (1a)-(1b). We also observe that formulation (5) is nonconvex in $q$ because of the constraints

$$
g_{i j}-\mu_{i j}=B_{i} q_{i}^{2}+B_{j} q_{j}^{2}-2 C_{i j} q_{i} q_{j}=\sum_{k \in K}\left(v_{i} u_{i k} q_{i}-v_{j} u_{j k} q_{j}\right)^{2} \quad \forall i<j \in A .
$$

We remark that a convex relaxation can be readily obtained by relaxing Eq. (6) to

$$
g_{i j}-\mu_{i j} \geq B_{i} q_{i}^{2}+B_{j} q_{j}^{2}-2 C_{i j} q_{i} q_{j} \quad \forall i<j \in A
$$

\section{Computational results}

We tested the SIP formulation (1a)-(1b) using native solver [14] which implements three types of discretization methods, but they could not solve most of the non-trivial instances $(n>2)$. Then, we tested our new formulation (5) of the ADP in $k$ dimensions on some 2D and 3D instances.

The set of 2D instances, already used in [15], is taken from [2]. It consists of circle instances where $n$ aircraft are placed on a circle of a given radius $r$, and non-circle instances where aircraft move along straight trajectories intersecting in $n_{c}$ conflict points.

The set of 3D instances is introduced in [12] - see the public repository https:// github.com/MartinaCerulli/SRADP - and it includes both sphere instances, where $n$ aircraft are placed on a sphere of a given radius $r$, and instances in which aircraft move along straight 3D trajectories (named non-sphere instances in Table 1), which intersect in at least $\frac{n}{2}$ conflict points.

For the $2 \mathrm{D}$ and the sphere instances the planned speed is $v_{i}=400 \mathrm{NM} / \mathrm{h}$ for each $i \in A$ and parameters $x_{i k}^{0}$ and $u_{i k}$ are given by

$$
u_{i 1}=\cos \left(\phi_{i}\right) \sin \left(\gamma_{i}\right), \quad u_{i 2}=\sin \left(\phi_{i}\right) \sin \left(\gamma_{i}\right), \quad u_{i 3}=\cos \left(\gamma_{i}\right), \quad x_{i k}^{0}=-r u_{i k}
$$

where $\gamma_{i}$ is the angle that the vector of the direction $u_{i}$ forms with the axis $k_{3}$ and $\phi_{i}$ is the angle between the projection of $u_{i}$ onto the $k_{1} k_{2}$-plane and the axis $k_{1}$. The bounds $q_{i}^{\min }$ and $q_{i}^{\max }$ are set to 0.94 and 1.03 respectively, following the weaker bounds proposed by the ERASMUS project [1].

We implemented the PP formulation (5) using the AMPL modeling language [16] and solved it with the global optimization solver Gurobi [17] ( $\mathrm{G}$ in the Table 1). For cases in which Gurobi exceeded its time-limit (set to 36000 seconds), we used a multistart algorithm (MS in Table 1), which performs 1000 calls to the IPOPT [18] local non-linear programming (NLP) solver from randomly sampled starting points. 
The headings of Table 1 are the following: $n$ number of aircraft; $r$ radius in NM of the circle or the sphere for 2D and 3D instances respectively; Literature Best obj is the best objective value found by each model; cpu computing time in seconds; slv solver used

In Table 1, for 2D instances we compare the results obtained by testing our PP reformulation on 2D instances with those that are obtained by solving the MINLP formulation proposed in [2] with the global solver Couenne [19] — Gurobi can't handle nonquadratic nonlinear constraints -, with default options but again with a time limit of $36000 \mathrm{sec}-$ onds. Whenever this time limit is reached, we report in italics lower and upper bound (inf when no feasible solution is found within the time limit) computed by Couenne.

The benchmarks for 3D instances are taken from the implementation of the approaches proposed in our working paper [12], where the ADP is formulated using Bilevel Programming and then reformulated into a single level non linear problem in three different ways. We report in the second column of Table 1 the best results among those obtained by solving the three NLP reformulations proposed in [12] with Couenne - Gurobi can't handle nonquadratic nonlinear constraints - , when we use Gurobi for solving formulation (5), or with the multistart algorithm when we use it for Probl.(5). Again, for the global solver Couenne we set a time limit of 36000 seconds, which is the same that we set for Gurobi. Whenever this time limit is reached, we report in italics lower and upper bound (inf when no feasible solution is found within the time limit) computed by the solver.

In all our tests, run on NEOS Server [20,21,22], we considered a time interval of $T=2$ hours and safety distance $d=5 \mathrm{NM}$.

The value of the objective function is always very low, given the tight speed variation bounds imposed by ERASMUS project $\left(q \in\left[q^{\min }, q^{\max }\right]\right)$. Best values are reported in bold for each instance. We can note that the formulation (5) is the best for most of the instances in terms of both solution quality and efficiency.

In particular, for 2D instances Couenne can find the global optimum only for small instances, namely those with $n \leq 5$; for the larger circle instances, it reaches the time limit providing only a lower bound $(L B)$, while for the non-circle ones, it provides a feasible solution too. On the contrary, with our approach we can always find an optimal solution within the time limit.

For 3D instances, when Couenne and Gurobi are used to solve NLP models proposed in [12] and formulation (5) respectively, Gurobi always outperforms Couenne, which cannot even find the global optimum for larger non-speric instances. When the multistart algorithm is used, for half of the instances the value found with our approach is the best, but we have no proof of global optimality. 
REFERENCES

Table 1: Numerical results

\begin{tabular}{|c|c|c|c|c|c|c|c|}
\hline \multicolumn{2}{|c|}{ Instances } & \multicolumn{3}{|c|}{ Literature } & \multicolumn{3}{|c|}{ Formulation (5) } \\
\hline$n$ & $r$ & Best obj/LB-UB & time $(\mathrm{s})$ & solver & obj & time $(\mathrm{s})$ & solver \\
\hline \multicolumn{8}{|c|}{ Circle } \\
\hline 2 & 100 & 0.002531 & 0.09 & Couenne & 0.002531 & 0.07 & G \\
\hline 3 & 200 & 0.001667 & 1.15 & Couenne & 0.001663 & 0.70 & G \\
\hline 4 & 200 & 0.004019 & 237 & Couenne & 0.004021 & 19.0 & G \\
\hline 5 & 300 & 0.003047 & 20698 & Couenne & 0.003049 & 46.1 & G \\
\hline 6 & 300 & $0.005463-i n f$ & Time Limit & Couenne & 0.006042 & 87.4 & G \\
\hline 8 & 400 & $0.000479-i n f$ & Time Limit & Couenne & 0.008247 & 34500 & G \\
\hline \multicolumn{8}{|c|}{ Non-circle } \\
\hline 6 & - & $0.000521-0.001254$ & Time Limit & Couenne & 0.001251 & 12.0 & $\mathrm{G}$ \\
\hline 7 & - & $0.000023-0.001617$ & Time Limit & Couenne & 0.001589 & 18.6 & G \\
\hline 7 & - & $0.000009-0.002147$ & Time Limit & Couenne & 0.001565 & 24.0 & G \\
\hline 8 & - & $0.002372-0.002384$ & Time Limit & Couenne & 0.002381 & 444 & G \\
\hline 10 & - & $0.000000-0.001543$ & Time Limit & Couenne & 0.001395 & 1472 & G \\
\hline \multicolumn{8}{|c|}{ Spheric } \\
\hline 2 & 100 & 0.002227 & 0.16 & Couenne & 0.002226 & 0.09 & G \\
\hline 3 & 200 & 0.001408 & 11.4 & Couenne & 0.001405 & 0.98 & G \\
\hline 4 & 200 & 0.003714 & 119 & Couenne & 0.003708 & 2.30 & $\mathrm{G}$ \\
\hline 5 & 300 & 0.002976 & 4890 & Couenne & 0.002943 & 51.1 & $\mathrm{G}$ \\
\hline 6 & 300 & 0.005320 & 67.5 & $\mathrm{MS}$ & 0.005847 & 59.1 & $\mathrm{MS}$ \\
\hline 7 & 500 & 0.002857 & 161 & MS & 0.002855 & 209 & MS \\
\hline 8 & 500 & 0.004566 & 672 & MS & 0.004513 & 104 & MS \\
\hline 9 & 500 & 0.006457 & 91.0 & MS & 0.006987 & 127 & MS \\
\hline 10 & 600 & 0.006333 & 443 & MS & 0.006393 & 161 & MS \\
\hline 12 & 700 & 0.008448 & 1727 & MS & 0.008380 & 222 & $\mathrm{MS}$ \\
\hline \multicolumn{8}{|c|}{ Non-spheric } \\
\hline 2 & - & 0.000305 & 0.16 & Couenne & 0.000304 & 0.33 & G \\
\hline 4 & - & 0.003283 & 188 & Couenne & 0.003282 & 1.72 & G \\
\hline 6 & - & 0.006004 & 6291 & Couenne & 0.006002 & 4.40 & G \\
\hline 8 & - & $0.000258-0.011705$ & Time Limit & Couenne & 0.011703 & 3.91 & G \\
\hline 10 & - & $0.000838-0.015025$ & Time Limit & Couenne & 0.015022 & 13.4 & G \\
\hline
\end{tabular}

\section{References}

[1] Erasmus: En route air traffic soft management ultimate system - final report ${ }^{1}$. Eurocontrol Experimental Centre, 2009.

[2] S. Cafieri and N. Durand. Aircraft deconfliction with speed regulation: New models from mixed-integer optimization. J. of Global Optimization, 58:613-629, 2014.

\footnotetext{
${ }^{1}$ ERASMUS deliverables are available at www. atm-erasmus.com
} 
[3] S. Cafieri and C. D'Ambrosio. Feasibility pump for aircraft deconfliction with speed regulation. Journal of Global Optimization, Springer Verlag, 71(3):501-515, 2018.

[4] L. Pallottino, E. Feron, and A. Bicchi. Conflict resolution problems for air traffic management systems solved with mixed integer programming. IEEE transactions on intelligent transportation systems, 3(1):3-11, 2002.

[5] C. Peyronne, A. R. Conn, M. Mongeau, and D. Delahaye. Solving air traffic conflict problems via local continuous optimization. European Journal of Operational Research, 241(2):502 - 512, 2015.

[6] A. Alonso-Ayuso, L. F. Escudero, and F. J. Martín-Campo. Exact and approximate solving of the aircraft collision resolution problem via turn changes. Transportation Science, 50:263-274, 2016.

[7] D. Rey and H. Hijazi. Complex number formulation and convex relaxations for aircraft conflict resolution. IEEE 56th Annual Conference on Decision and Control, pages $88-93,2017$.

[8] A. Bahr, J. Leonard, and M. Fallon. Cooperative localization for autonomous underwater vehicles. International Journal of Robotics Research, 28(6):714-728, 2009.

[9] C. Lizon, C. D’Ambrosio, L. Liberti, M. Le Ravalec, and D. Sinoquet. A mixedinteger nonlinear optimization approach for well placement and geometry. In Proceedings of the 14th European Conference on the Mathematics of Oil Recovery, volume XIV of ECMOR, page A38, Houten, 2014. EAGE.

[10] L. Wang and F. Guo. Semidefinite relaxations for semi-infinite polynomial programming. Computational Optimization and Applications, 58:133-159, 2014.

[11] P. Tabaghi, I. Dokmanić, and M. Vetterli. Kinetic Euclidean distance matrices. IEEE Transactions on Signal Processing, 68:452-465, 2020.

[12] M. Cerulli, C. D’Ambrosio, L. Liberti, and M. Pelegrín. Detecting and solving aircraft conflicts using bilevel programming. Working paper, 2020. Available at https: //hal .archives-ouvertes.fr/hal-02869699v3.

[13] Y. Xu, W. Sun, and L. Qi. On solving a class of linear semi-infinite programming by SDP method. Optimization, 64(3):603-616, 2015.

[14] A. Vaz, E. Fernandes, and M. Gomes. Nsips version 2.1 nonlinear semi-infinite programming solver.

[15] M. Cerulli, C. D'Ambrosio, and L. Liberti. Flying safely by bilevel programming. Advances in Optimization and Decision Science for Society, Services and Enterprises: ODS, Genoa, Italy, September 4-7, 2019, 3:197-206, 2019. Available at https: //hal.archives-ouvertes.fr/hal-02869682.

[16] R. Fourer, D. M. Gay, and B. W. Kernighan. AMPL: A Modeling Language for Mathematical Programming. Cengage Learning, 2002. 
REFERENCES

[17] LLC Gurobi Optimization. Gurobi optimizer reference manual, 2020.

[18] A. Wächter and L. Biegler. On the implementation of a primal-dual interior point filter line search algorithm for large-scale nonlinear programming. Mathematical Programming, 106:25-57, 2006.

[19] P. Belotti, J. Lee, L. Liberti, and A. Wächter. Branching and bounds tightening techniques for non-convex minlp. Optimization Methods and Software, 24(4-5):597634, 2009.

[20] J. Czyzyk, M. P. Mesnier, and J. J. Moré. The NEOS server. IEEE Journal on Computational Science and Engineering, 5(3):68 -75, 1998.

[21] E. D. Dolan. The NEOS server 4.0 administrative guide. Technical Memorandum ANL/MCS-TM-250, Mathematics and Computer Science Division, Argonne National Laboratory, 2001.

[22] W. Gropp and J. J. Moré. Optimization environments and the NEOS server. In Approximation Theory and Optimization, pages 167 - 182. Cambridge University Press, 1997. 